\title{
Magnetic Field Shaping Technique for HF-RFID and NFC Systems
}

\author{
Pinitnai SITTITHAI ${ }^{1}$, Kittisak PHAEBUA ${ }^{1}$, Titipong LERTWIRIYAPRAPA ${ }^{1}$, \\ Prayoot AKKARAEKTHALIN ${ }^{2}$
}

\author{
${ }^{1}$ Industrial Electric and Control System Research Center, Dept. of Teacher Training in Electrical Engineering, \\ Faculty of Technical Education, \\ ${ }^{2}$ Dept. of Electrical and Computer Engineering, Faculty of Electrical Engineering, \\ King Mongkut's University of Technology North Bangkok. 1518 Pracharat 1 Rd., Bangkok 10800, Thailand
}

pinitnai@gmail.com, kittisak.p@fte.kmutnb.ac.th, titipong.1@fte.kmutnb.ac.th, prayoot.a@eng.kmutnb.ac.th

Submitted June 22, 2018 / Accepted January 4, 2019

\begin{abstract}
In this paper, the magnetic field shaping technique using the magnetic field repeater for high frequency radio frequency identification (HF-RFID) system and nearfield communications (NFC) system is proposed. This technique aims to improve the communication area between $H F-R F I D$ or NFC reader and passive tag by changing the magnetic field distribution over the original HF-RFID or $N F C$ reader antenna. The multiple rectangular loop resonators cooperating with low loss air variable capacitors are employed to construct the proposed magnetic field repeater. The proposed magnetic field repeater is installed on top of the original HF reader antenna without any direct connection to the original reader antenna. The $H F-$ $R F I D$ reader for laundry application is chosen as an example to prove the concept and verify the performance. We found that the communication area between the tag and off-the-shelf HF-RFID reader is improved. The proposed technique is useful for antenna improvement of off-the-shelf HF-RFID system in many applications such as laundry application, product tacking, etc. where a short range with wide communication area are required.
\end{abstract}

\section{Keywords}

High frequency (HF) radio frequency identification (RFID), magnetic field repeater, HF-RFID reader antenna, magnetic resonance technique

\section{Introduction}

High frequency radio frequency identification (HFRFID) systems are increasingly used for a short-range application because they are low cost and acceptable performance in term of detected distance for many applications [1] such as a smart card system, product tracking, money payment, etc. HF-RFID system is an item identification system employing a near-field electromagnetic (EM) coupling for both communication and power transfer wire- lessly. The passive HF-RFID tag receives the EM energy from HF-RFID reader to operate the IC-chip and sends back an identification data to HF-RFID reader [1]. Moreover, near-field communications system (NFC) is a subsequent system of HF-RFID technology. NFC was developed from HF-RFID technology both operating at the same frequency of $13.56 \mathrm{MHz}$ [2]. NFC is proposed for the data exchange applications with higher data security such as in mobile payment applications. NFC technology is usually installed on portable devices such as mobile phones, tablets, smart watches, etc. The communication mode of NFC is peer-to-peer communication [3], [4]. Generally, passive HF-RFID and passive NFC systems both employ the wireless power transfer (WPT) technology for electrical power transmission between reader and passive tag [5]. The electrical power is transferred wirelessly to the tag via the free space. The passive HF-RFID and NFC systems both employ the highly quality factor $(\mathrm{Q})$ magnetic resonant coupling technique [5-7], which is more efficient than the classical magnetic induction method in term of communication distance and misalignment between reader and tag antennas [5]. At the transmitter (Tx), a single loop antenna is usually employed to generate and receive the magnetic field as shown in Fig. 1(a). The capacitor is employed to tune the resonance frequency $f_{\mathrm{r}}$ which can be written as $f_{\mathrm{r}}=1 / 2 \pi \sqrt{L C}$. However, the disadvantages of the conventional (off-the-shelf) HF-RFID single loop transmitting antenna are narrow reading area problem and low performance in the term of an arbitrary HF-RFID tag orientations [2], [3]. Single loop transmitting antenna generates maximum magnetic field strength at the center of the loop. The magnetic field strength decays rapidly along the horizontal $x y$-plane. The dominant magnetic field component $B_{z}$ is in a normal direction to the loop (z-axis) as shown in Fig. 1(a). At receiver ( $R x)$, the maximum receiving power is occurred when the tag orientation is in the same direction as a magnetic field component ( $z$-axis). Those reasons lead to low performance in the case of an arbitrary HF-RFID tag orientations and narrow reading area (misalignment problem). 


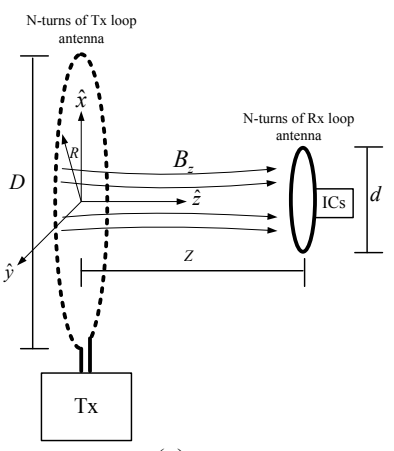

(a)

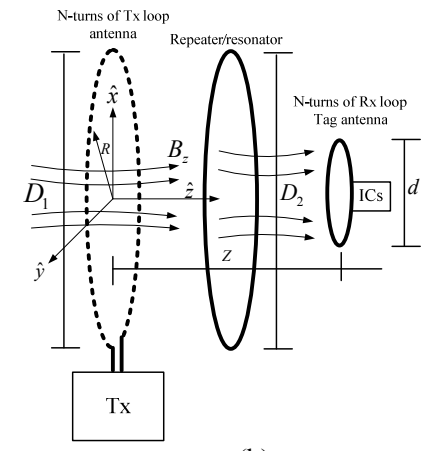

(b)

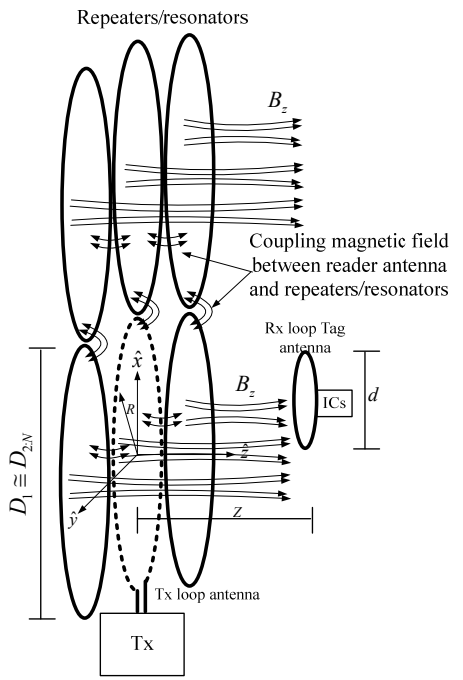

(c)

Fig. 1. (a) Passive HF-RFID and passive NFC systems diagram. (b) Axial alignment repeater. (c) Proposed horizontal alignment repeater.

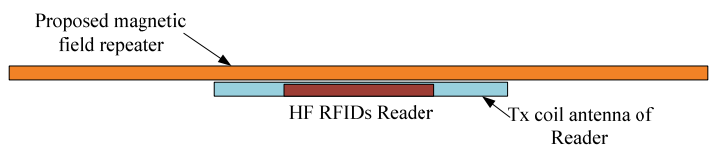

(a)

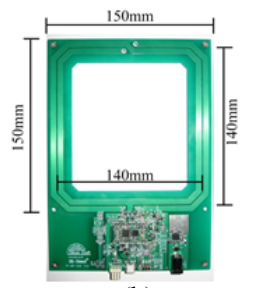

(b)

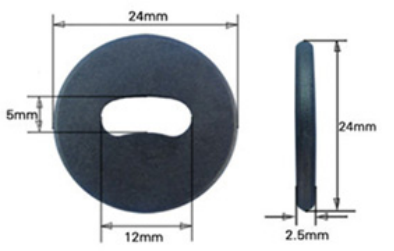

(c)
Fig. 2. (a) Cross section view of the proposed magnetic field repeater on an off-the-shelf HF-RFID reader. (b) Offthe-shelf HF-RFID reader (model: Pi-931-XA5) [15]. (c) RFID laundry tag ISO15693, I-code-SLI.

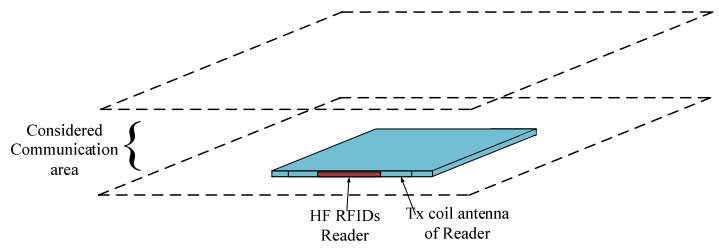

Fig. 3. Considered communication area $\left(500 \times 380 \mathrm{~mm}^{2}\right)$ on an off-the-shelf HF-RFID reader.
In the literature work [5], the power transfer efficiency and maximum distance depend on Tx and Rx antennas size and orientations. In the case of the $\mathrm{Tx}$ and $\mathrm{Rx}$ antennas are identical and perfectly aligned, bigger $\mathrm{Tx}$ and $\mathrm{Rx}$ coil provide larger maximum distance than smaller coil antennas. The maximum distance in the case of an acceptable efficiency $(50 \%)$ is around one times of coil diameter $D$. In the case of the Rx antenna is smaller than Tx antenna $(d / D<1)$ as shown in Fig. 1(a), the maximum power transfer efficiency decreases as a function of $d / D$ ratio where $d$ denotes Rx diameter and $D$ denotes Tx diameter. $d / D=1$ is the best case [5].

In the WPT system [6-10], several magnetic field shaping techniques are proposed to solve these problems. Transferred power in case of the misalignment and perfect alignment cases can be improved by using the magnetic materials to shape a magnetic flux density [7]. However, the magnetic materials produce magnetic loss and also increase the cost, size, weight and complexity of the system. Moreover, the antenna shapes also directly affect the magnetic field components and transferred power. Conventionally, the circular and rectangular antennas are usually employed in a typical wireless power transfer, NFC and HF-RFID systems [1-11]. The magnetic field distributions of both circular and rectangular antennas are very similar.

To improve the distance of wireless power transfer in the axial direction, a study on magnetic field repeater in wireless power transfer was present in [12]. The intermediate resonators (repeaters) are installed between the transmitter and the receiver to increase the distance of wireless power transfer. However, the Rx antenna size is the same as the Tx and repeater size. Several techniques in [12] can be applied for read range improvement of HF-RFID application where the repeaters are in the axial alignment case as the concept in Fig. 1(b) shows. Unfortunately, the study of the horizontal alignment of repeaters was not focused. Also, only 2 repeaters were studied. In contrast, to improve the wide area of wireless power transfer, the switchable Tx coil array antenna for free positioning of Rx was presented in $[5-8]$ and non-switchable array antenna was presented in [13-15]. The presented methods [5] are implemented to solve misalignment problem. The multiple Tx coils are employed to construct the coil array antenna. The multiple Tx coils are connected directly to the transmitter by using RF switches. The guided positioning was performed by using additional localization system. The multiple Tx coils will be scanned to find the receiver location and operate three overlapped Tx coils at the receiver position [5]. However, the system is very complicated and high cost. The direct connection between transmitter and each multiple $\mathrm{Tx}$ coils are required.

In addition, the multiple power repeater coil antennas are presented in [13-15]. Power repeater antennas for a portable device of wireless battery charging system are presented [13]. The charging distance is increased horizontally by using two identical repeater antennas. It is located in the same direction. The Tx antenna and repeaters 
are identical. Maximum distance in the case of $50 \%$ receiving power was studied. The optimum distribution of magnetic field over a considered area was ignored. The rule of thumb is that, the suitable spacing between each power repeaters and Tx antenna is around 1.05 times of repeater antenna diameter $\left(1.05 D_{\mathrm{r}}\right)$ measured from center to center of power repeaters. The power repeater antennas are not overlapped with each other. Moreover, the passive booster for improving magnetic coupling of metal mounted proximity range HF-RFIDs was presented in [14]. The single passive booster resonator contains two coils connected in parallel to each other without capacitor. The magnetic coupling between the small metal mounted in HF-RFIDs and the NFC coil are improved. However, only maximum charging distance in a single direction was focused. The optimum distribution of magnetic field was ignored. The power repeater is not overlapped with each other. Moreover, the multiple loop power repeater is being studied in [15]. The preliminary study of multiple repeater antennas for high frequency (HF) RFID reader in [15] is illustrated that the magnetic field distribution can be shaped by using the multiple repeater antennas. However, the simulation and experimental studies in [15] is not completed. In this paper, the case study in [15] is rigorously investigated and compared with the off-the-shelf model.

This paper presents a magnetic field shaping technique by using magnetic field repeater for HF-RFID system and NFC systems. The HF-RFID for laundry application is chosen to illustrate the performance of the proposed magnetic field repeater. The HF-RFID tag is attached on clothes and put on the reader to collect the data. The laundry application requires wide communication area on the reader surface. The misalignment of RFID tag on the cloth can be a major problem. The proposed magnetic field repeater is installed on top of the conventional HF-RFID reader antenna without any direct connection to improve the reading area as shown in Fig. 2(a). The electrical power is transferred via the free space by employing the magnetic resonant coupling technique. The magnetic field is stored in the proposed magnetic field repeater and repeats to receiver (tag). The magnetic field distribution over the considered area is changed depending on a configuration of multiple rectangular loop resonators inside the proposed magnetic field repeater. The HF-RFID system operating at $13.56 \mathrm{MHz}$ with ISO 15693 (I-code) standard is employed to illustrate the performance of the proposed magnetic field repeater. An example of off-the-shelf HF-RFID reader is shown in Fig. 2(b) [16]. Considered communication area $\left(500 \times 380 \mathrm{~mm}^{2}\right)$ over the original HF-RFID reader of laundry application is shown in Fig. 3.

\section{Magnetic Field Repeater Design}

\subsection{Selected Rectangular Loop Resonator}

Considering a conventional circular loop antenna [17], the magnetic field can be approximated as

$$
B_{\mathrm{z}}=\frac{\mu_{0}}{4 \pi} \frac{2 \pi N R^{2} I}{\left(Z^{2}+R^{2}\right)^{3 / 2}}
$$

where $B_{\mathrm{z}}$ denotes the magnetic field $\left(\mathrm{Wb} \cdot \mathrm{m}^{-2}\right) . R$ and $Z$ denote the radius of the loop and observation point distance normal to the loop, respectively. The magnetic field depends on the feeding current $I$, loop radius and number of wire turns $N$. It is found that the magnetic field is decreased as a function of axial distance $Z$ where current $I$, number of wire turns $N$ and $R$ are fixed. Approximately, the magnetic field is decreased along the axial distance as a function of $Z^{-3}$.

The quality factor $Q$ of the Tx and Rx loops plays a key role of the magnetic resonant coupling technique and affect the power transfer efficiency [5]. Higher $Q$ factor provides more power transfer efficiency. The $Q$ factor can be calculated as

$$
Q(\omega)=\omega \times \frac{\text { Maximum Stored Energy }}{\text { Power Loss }} .
$$

Equation (2) illustrates that lower loss in the loop provides higher $Q$ factor if we assume that the maximum stored energy in the loop is fixed. Alternatively, $Q$ factor of the loop can be written as

$$
Q=\frac{\omega L}{R_{\text {loop }}}
$$

when $L$ is an inductance $(\mathrm{H})$ of a single loop antenna. The $R_{\text {loop }}$ denotes the total resistance of a single loop antenna. Generally, in the case of conventional circular helical coil antenna with loop radius $a$, wire radius $b$, and wire spacing $2 c$ in [17], $R_{\text {loop }}$ can be calculated as

$$
R_{\text {loop }}=\frac{N a}{b} R_{\mathrm{s}}\left(\frac{R_{\mathrm{p}}}{R_{0}}+1\right)
$$

where $R_{s}=\sqrt{\omega \mu_{0} / 2 \sigma}$ denotes surface impedance of a conductor. $R_{\mathrm{p}}$ denotes an ohmic resistance due to the proximity effect. The proximity effect is occurred when the wire of each turns of the loop closed each other. More spacing provides lower $R_{\mathrm{p}} . R_{0}=N R_{\mathrm{s}} /(2 \pi b)$ is ohmic skin effect resistance per unit length $(\mathrm{ohms} / \mathrm{m})$. As mentioned, the electrical properties of rectangular loop are similar to the circular loop type. Therefore, $R_{\text {loop }}$ of rectangular loop with $N$ turns, width $W$, wire radius $D_{\text {wire }} / 2$ and wire spacing $S=2 c$ as shown in Fig. 4 can be approximated by using (3) and (4) by replacing $a \cong W / 2$ and $b=D_{\text {wire }} / 2 . R_{\mathrm{p}}$ is relatively high comparing with $R_{0}$. In [17], the ratio of $R_{\mathrm{p}} / R_{0}$ value decreases as a function $c / b$ ratio $\left(S / D_{\text {wire }}\right)$ increases. The ratio of $S / D_{\text {wire }}$ should be greater than factor of 3 to provide low $R_{\mathrm{p}} / R_{0}$ ratio where other parameters are fixed. Thus, the wire spacing $S$ of each turn should be greater than wire diameter around 3 times $\left(S \geq 3 D_{\text {wire }}\right)$. More wire spacing $S$ provides low $R_{\text {loop }}$ resistance due to low proximity effect. Also, the number of wire turns $N$ affects directly to $R_{\text {loop }}$ and the capacitance value of tuning capacitor which is available in market.

In this work, the rectangular loop resonator is chosen to construct the magnetic field repeater. The magnetic field 
in the axial of the rectangular loop is similar to the circular loop type [18]. The parameters $W, S, N$ and $D_{\text {wire }}$ denote the width of loop, spacing of wire, number of wire turns and diameter of wire, respectively. The rectangular loop resonators provide high $Q$ factor when the total resistance of the loop $R_{\text {loop }}$ is minimum. The width of loop $W=$ $150 \mathrm{~mm}$ is chosen due to the fact that the maximum magnetic field coupling between two coils (reader and repeater) of the magnetic field resonance technique is occurred when the diameter ratio between both antennas $D_{2} / D_{1}$ is approached to unity [5] as shown in Fig. 1(b). All parameters are shown in Tab. 1 . The combination of multiple rectangular loop resonators provides optimum magnetic field distribution than a single large loop resonator. The wire spacing around three times of diameter $3 D_{\text {wire }}$ is chosen to minimize the total resistance of the loop. The number of wire turns $(N=4)$ is chosen to minimize total resistance $R_{\text {loop }}$ and provide suitable inductance value for available capacitor. Wire diameter $D_{\text {wire }}$ of $2 \mathrm{~mm}$ is selected. The low loss air variable capacitor $(20-126 \mathrm{pF})$ is employed to tune the resonance frequency of $13.56 \mathrm{MHz}$. The normalized magnitude of $S_{21}(\mathrm{~dB})$ of the resonance frequency characteristics of the rectangular and circular loops are illustrated in Fig. 4(b). It is measured by using network analyzer with small loop probes as the measurement procedure in Fig. 4(c) shows. The frequency characteristics of rectangular and circular loops are very similar.

\begin{tabular}{|c|c|c|}
\hline Symbol & Quantity & Dimension $(\mathrm{mm})$ \\
\hline$W$ & Width of loop & 150 \\
\hline$S$ & Spacing of wire & 6 \\
\hline$D_{\text {wire }}$ & Diameter of wire & 2 \\
\hline$N$ & Number of wire turns & 4 \\
\hline
\end{tabular}

Tab. 1. Optimum parameters of selected rectangular loop resonators.

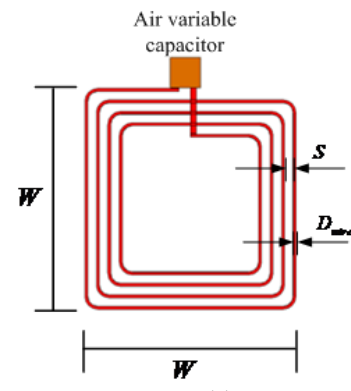

(a)

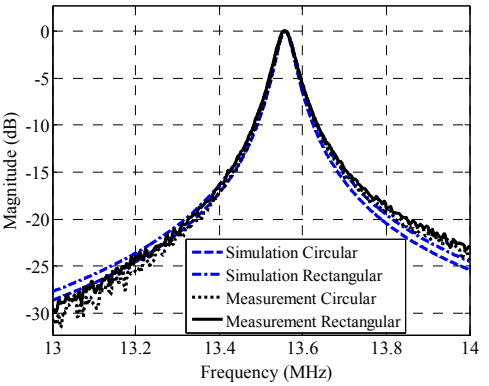

(b)

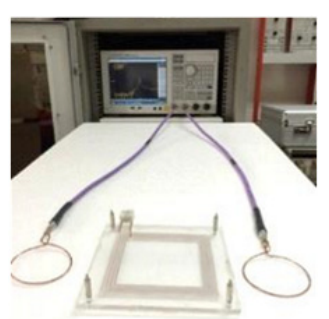

(c)

Fig. 4. (a) Selected rectangular loop resonator. (b) Normalized magnitude of $\left|\mathrm{S}_{21}\right|(\mathrm{dB})$ of a resonance frequency characteristic of rectangular and circular loop antennas. (c) Measurement of magnitude of $\left|\mathrm{S}_{21}\right|(\mathrm{dB})$ by a small loop probe.

\subsection{Optimization Procedure and Magnetic Field Shaping Investigation.}

Unlike the previous works [12-14], an improvement of magnetic field distribution over a considered area will be focused in this paper. The magnetic field repeaters are employed to store and repeat the magnetic field between the reader antenna and tag as shown in Fig. 1(c). In simulation, the current excitation is required. However, it is difficult to find the actual current pass though the antenna of the complex reader circuit on a printed circuit board (PCB). The differential probe is required to measure voltage and impedance of the RFID reader, which is located on PCB. It is noted that many parasitic elements on PCB affect the actual impedance and real current flow pass though the antenna. To avoid the complexity in the simulation, the normalized excitation current of $1 \mathrm{~A}$ is employed for every simulation case. The proposed design procedure by using the simulation together with the measurement will be explained in this section.

Generally, the RFID tag will be operated under the condition of $H \geq H_{\min }$ where $H$ denotes the magnetic field strength at the tag location and $H_{\text {min }}$ denotes the minimum magnetic field strength of HF-RFID laundry tag. In the optimization procedure, the minimum magnetic field strength $H_{\min }$ is the criterion. The magnetic field at observation points in the considered area is focused. The goal of optimization is the maximum total detectable area over the HF-RFID reader with $H \geq H_{\min }$ condition. The position of each rectangular loop resonator inside the proposed magnetic field repeater will be optimized.

As mentioned above, the actual $H_{\text {min }}$ value depends on the model of RFID tags. It is difficult to measure the actual $H_{\min }$ of the tag. In this paper, the equivalent $H_{\min }$ is approximated by using the measurement procedure together with the simulation. The procedures to estimate the equivalent $H_{\min }$ of HF-RFID laundry tag in this paper are as follows:

- Step 1: In measurement, off-the-shelf HF-RFID reader antenna is employed as shown in Fig. 2(b). The HF-RFID laundry tag as shown in Fig. 2(c) is employed to find the maximum detectable location $R_{\max }$. The tag position will be moved away from the reader and stop at last detectable location $R_{\max }$ as shown in Fig. 5(a). It is found that $R_{\max }$ is $150 \mathrm{~mm}$

- Step 2: In the simulation, off-the-shelf HF-RFID reader antenna configuration is precisely modeled in CST-Microwave Studio [19] as shown in Fig. 5(b). In this case, the $1 \mathrm{~A}$ current excitation from the reader is employed. The magnetic probe is modeled at $R_{\max }$ from the measurement step 1 . The equivalent $H_{\min }$ can be approximated. The simulated magnetic field strength $(\mathrm{A} / \mathrm{m})$ versus observation location is illustrated in Fig. 5(c).

Approximately, it is found that the equivalent magnetic field strength around $1.6 \mathrm{~A} / \mathrm{m}$ (yellow color) at $R_{\max }$ 
of $150 \mathrm{~mm}$ is suitable to represent the equivalent $H_{\min }$ for the optimization procedure in every case. Note that $H_{\min }$ of $1.6 \mathrm{~A} / \mathrm{m}$ is not an actual value of magnetic field strength of the tag. It is relative to the source excitation in simulation setup. It may be not accurate for other models of RFID reader. The studies of magnetic field shaping of two rectangular loop resonators are illustrated in Fig. 6. Figure 6(a) shows simulated magnetic field distribution in the case of an off-the-shelf HF-RFID reader antenna (only RFID reader), Figures 6(b) to (d) illustrate the spacing between rectangular loop resonators of $100 \mathrm{~mm}, 0 \mathrm{~mm}$ and overlapped, respectively. In simulation, by using equivalent $H_{\text {min }}$ of $1.6 \mathrm{~A} / \mathrm{m}$ criteria, we found that the overlapped case as shown in Fig. 6(d) provides larger detectable area than other cases. In this case, the rectangular loop resonators with some overlapped part are chosen to construct the proposed magnetic field repeater in the next section.

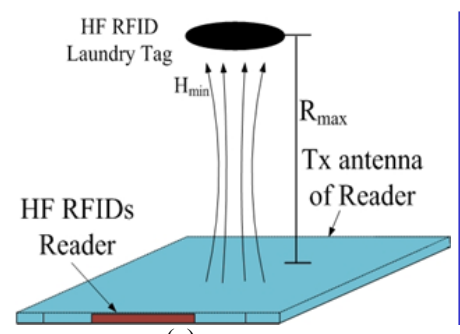

(a)

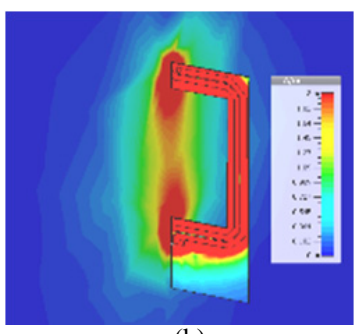

(b)

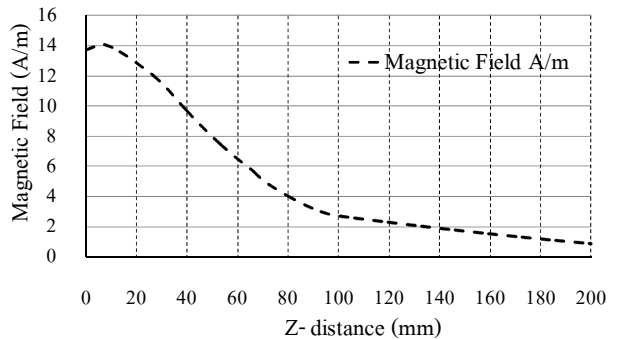

$\mathrm{Z}$ - distance $(\mathrm{mm})$

(c)

Fig. 5. Measurement procedure of approximated $H_{\min }$ : (a) find $R_{\max }$, (b) simulated magnetic field distribution (off-theshelf HF-RFID reader antenna), (c) simulated magnetic field strength $(\mathrm{A} / \mathrm{m})$ versus observation locations.

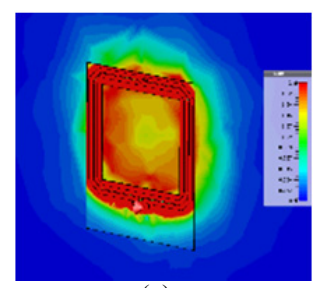

(a)

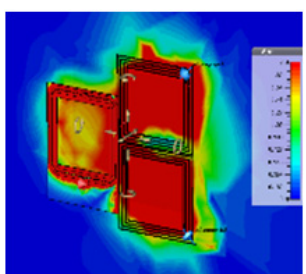

(c)

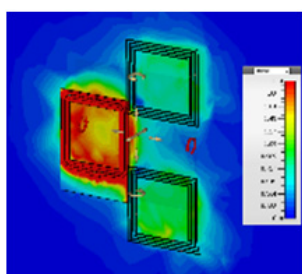

(b)

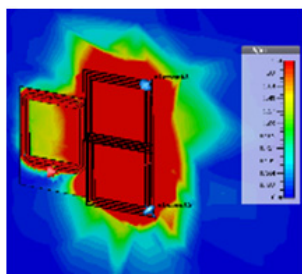

(d)
Fig. 6. Magnetic field distribution: (a) without resonator, (b) two rectangular loop resonators with spacing of $100 \mathrm{~mm}$, (c) spacing of $0 \mathrm{~mm}$, (d) overlapped case.

\subsection{Proposed Magnetic Field Repeater}

The proposed magnetic field repeater is shown in Fig. 7(a) while the previous model [15] is shown in Fig. 7(b). The proposed magnetic field repeater consists of multiple rectangular loop resonators. The width of the loop is fixed. Five rectangular loop resonators are chosen to provide optimum magnetic field distribution over the considered area (application requirement). $W=150 \mathrm{~mm}$, $S=6 \mathrm{~mm}$ and $N=4$ are chosen under the condition of minimum total resistance $R_{\text {loop }}$ and suitable for an available capacitor in the market. The rest parameters are optimized by using CST Microwave Studio program [19]. $O_{\mathrm{s}}, O_{\text {sy }}$ and $O_{\text {sx }}$ denote the overlapped area and spacing of rectangular loop resonators, respectively. The optimum magnetic field distribution over the reader surface of $500 \times 380 \mathrm{~mm}^{2}$ is the goal of optimization $H \geq H_{\min } \geq 1.6 \mathrm{~A} / \mathrm{m}$. The equivalent minimum magnetic field strength $H_{\min }$ of $1.6 \mathrm{~A} / \mathrm{m}$ criterion is employed to find the maximum detectable area (including layers 1 to 7 as shown in Fig. 9). The optimum parameters are shown in Tab. 2 while the optimum designed parameters of the previous model [15] are shown in Tab. 3.

The simulated results of magnetic field distribution are illustrated in Fig. 8. The simulated magnetic field distribution of the proposed magnetic field repeater is shown in Fig. 8(a). The magnetic field spread out from the center

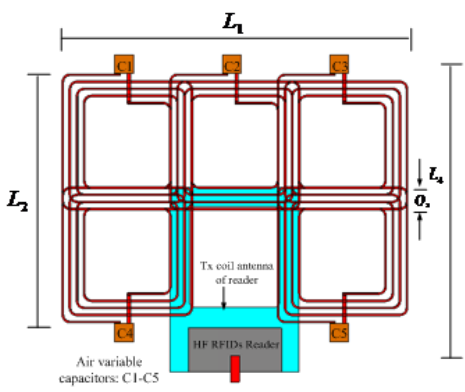

(a)

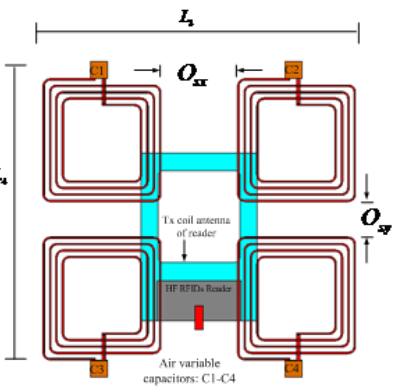

(b)
Fig. 7. (a) The proposed magnetic field repeater, (b) previous model [15].

\begin{tabular}{|c|c|c|}
\hline Symbol & Quantity & Dimension (mm) \\
\hline$W$ & Width of loop & 150 \\
\hline$S$ & Spacing of wire & 6 \\
\hline$D_{\text {wire }}$ & Diameter of wire & 2 \\
\hline$N$ & Number of wire turns & 4 \\
\hline$O_{\mathrm{s}}$ & Overlap spacing & 26 \\
\hline$L_{1}$ & Length & 408 \\
\hline$L_{2}$ & Width & 336 \\
\hline
\end{tabular}

Tab. 2. Optimum parameters of the proposed magnetic field repeater.

\begin{tabular}{|c|c|c|}
\hline Symbol & Quantity & Dimension (mm) \\
\hline$O_{\text {sy }}$ & y-spacing & 60 \\
\hline$O_{\text {sx }}$ & x-spacing & 140 \\
\hline$L_{3}$ & Length & 408 \\
\hline$L_{4}$ & Width & 360 \\
\hline
\end{tabular}

Tab. 3. Optimum parameters of the previous model [15]. 


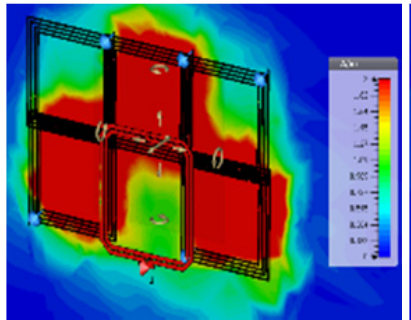

(a)

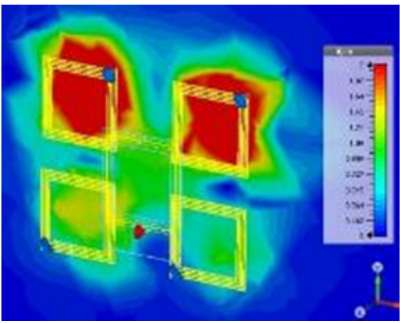

(b)

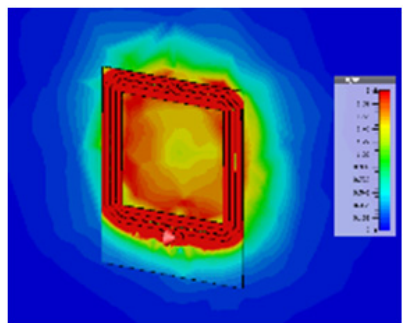

(c)

Fig. 8. Simulated magnetic field distribution of (a) the proposed magnetic field repeater, (b) the previous model, (c) conventional HF-RFID reader antenna (model: Pi-931-XA5).

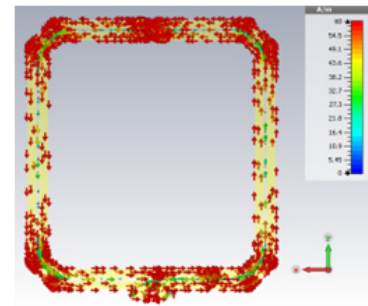

(a)

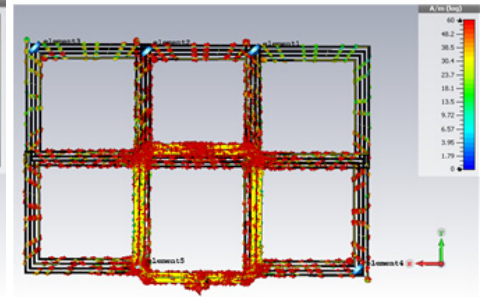

(b)
Fig. 9. Simulated current distribution at $13.56 \mathrm{MHz}$ : (a) without resonator, (b) overlapped resonator case.

of the original reader antenna due to the proposed magnetic field repeater. The simulated result of magnetic field distribution of the previous model is shown in Fig. 8(b). Four rectangular loop resonators are employed for comparison. However, the magnetic field strength is lower than the proposed model. Finally, the simulated result of magnetic field distribution of an off-the-shelf HF-RFID reader antenna model Pi-931-XA5 is shown in Fig. 8(c). The magnetic field is very strong at the center of the reader antenna. However, the magnetic field is rapidly decreased as function of horizontal distance. The study shows that the overlapped multiple rectangular loop resonators provide optimum magnetic field distribution inside the considered area and cover wider area than the off-the-shelf HF-RFID reader and previous model [15].

Figure 9 shows the simulated current distribution at 13.56 MHz. Without resonator case is shown in Fig. 9(a). The overlapped multiple rectangular loop resonators are shown in Fig. 9(b). The magnetic field from the reader antenna excites each resonator and regenerates the surface current at resonators. Very strong surface current at each resonator provides strong magnetic field in the considered area. The magnetic field distribution of the original reader is changed by using the proposed repeater.

\section{Experimental Results}

The measurement of reading (communication) area of the proposed magnetic field repeater is performed by measuring the communication status between the tag and HF-RFID reader. The RFID tag of ISO15693 I-code-SLI standard for laundry application as shown in Fig. 2(c) is employed to evaluate the performance of the proposed magnetic field repeater. The off-the-shelf HF-RFID reader model Pi-931-XA5 [16] is employed. The RFID tag will be moved over the testing surface of layers 1 to 6 on the reader antenna with the proposed repeater as shown in Fig. 10. The sampling spacing is $2.5 \mathrm{~cm}$ with 20 sampling horizontal points and 15 vertical points. Six layers are measured over the reader antenna with the proposed repeater. It is noted that dark blue color denotes an undetectable status. Light blue, blue, green, yellow, red and dark red colors represent a detectable status until layers of $10 \mathrm{~mm}, 30 \mathrm{~mm}, 60 \mathrm{~mm}, 90 \mathrm{~mm}, 120 \mathrm{~mm}$ and $150 \mathrm{~mm}$, respectively. The total sampling points of each layer are 300 points. The total testing points of each case are 1800 points (6 layers).

In this experiment, three cases are considered namely, case 1: the proposed magnetic field repeater, case 2: the previous model [15] and case 3: the off-the-shelf HF-RFID reader antennas as shown in Fig. 11. It is obvious that the experimental result in the case 1 , the proposed magnetic field repeater provides a detectable area of $1198.08 \mathrm{~cm}^{2}$, $645.12 \mathrm{~cm}^{2}, \quad 443.52 \mathrm{~cm}^{2}, \quad 190.08 \mathrm{~cm}^{2}$ in the layers of $10 \mathrm{~mm}, 30 \mathrm{~mm}, 60 \mathrm{~mm}$ and $90 \mathrm{~mm}$, respectively as shown

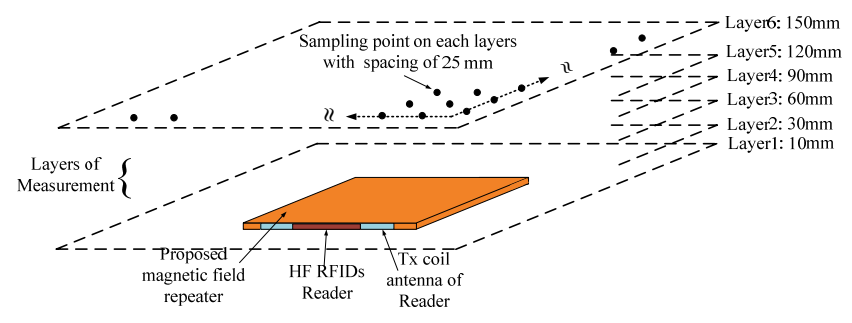

Fig. 10. Considered communication area and measurement procedure.

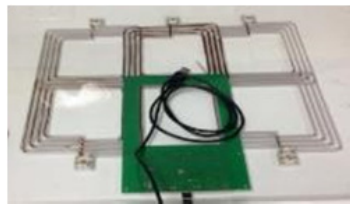

(a)

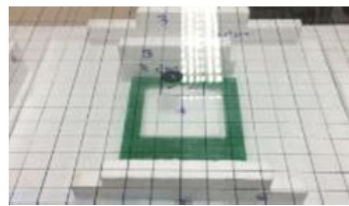

(c)

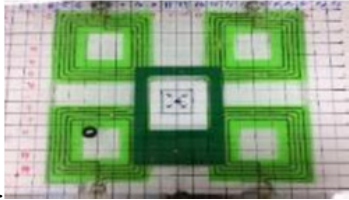

(b)

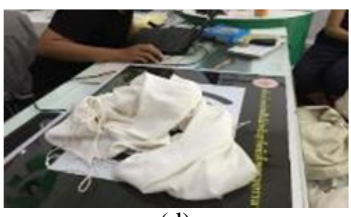

(d)
Fig. 11. Prototypes of (a) the proposed magnetic field repeater, (b) the previous model [15], (c) the conventional HFRFID reader antenna (model: Pi-931-XA5), (d) actual situation experiment. 


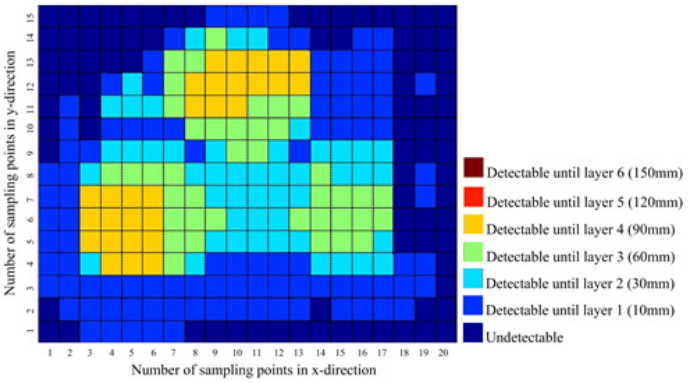

(a)

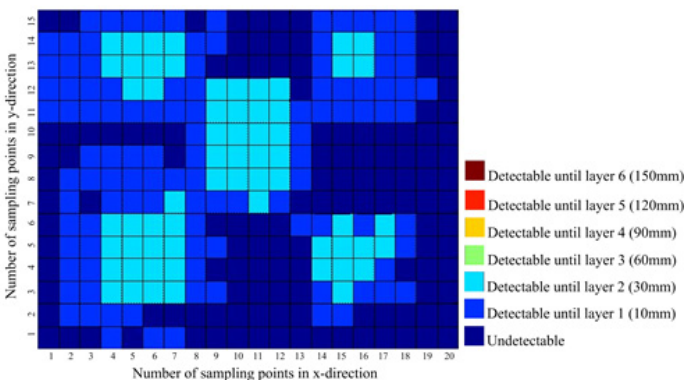

(b)

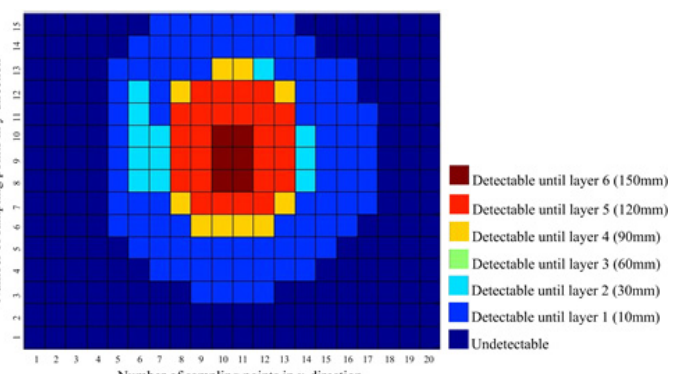

(c)

Fig. 12. Experimental results of the detected area: (a) the proposed magnetic field repeater, (b) the candidate model [15], (c) the conventional HF-RFID reader antenna.

\begin{tabular}{|c|c|c|c|}
\hline Models & $\begin{array}{l}\text { Measurement } \\
\text { layers (mm) }\end{array}$ & $\begin{array}{l}\text { Detectable } \\
\text { area }\left(\mathrm{cm}^{2}\right)\end{array}$ & $\begin{array}{c}\text { Gains and losses } \\
\text { of detectable } \\
\text { area }\left(\mathrm{cm}^{2}\right) \\
\text { compared with } \\
\text { off-the-shelf } \\
\text { RFID reader } \\
\end{array}$ \\
\hline \multirow{7}{*}{$\begin{array}{c}\text { Proposed } \\
\text { magnetic } \\
\text { field repeater }\end{array}$} & 10 & 1198.08 & 397.44 \\
\hline & 30 & 645.12 & 374.4 \\
\hline & 60 & 443.52 & 161.28 \\
\hline & 90 & 190.08 & -51.84 \\
\hline & 120 & 0 & -184.32 \\
\hline & 150 & 0 & -34.56 \\
\hline & Sum & $2,475.8$ & \\
\hline \multirow{7}{*}{$\begin{array}{c}\text { Previous } \\
\text { model [15]. }\end{array}$} & 10 & 961.92 & 161.28 \\
\hline & 30 & 380.16 & 109.44 \\
\hline & 60 & 0 & -282.24 \\
\hline & 90 & 0 & -241.92 \\
\hline & 120 & 0 & -184.32 \\
\hline & 150 & 0 & -34.56 \\
\hline & Sum & 1,342 & \\
\hline \multirow{7}{*}{$\begin{array}{l}\text { Off-the-shelf } \\
\text { HF-RFID } \\
\text { reader } \\
\text { (model: Pi- } \\
\text { 931-XA5) }\end{array}$} & 10 & 800.64 & - \\
\hline & 30 & 270.72 & - \\
\hline & 60 & 282.24 & - \\
\hline & 90 & 241.92 & - \\
\hline & 120 & 184.32 & - \\
\hline & 150 & 34.56 & - \\
\hline & Sum & $1,814.4$ & \\
\hline
\end{tabular}

Tab. 4. Experimental results. in Fig. 12(a) and results shown in Tab. 4. It is better than the off-the-shelf HF-RFID reader as shown in Fig. 12(c) and results shown in Tab. 4. In the case 2, the detectable areas of previous model [15] are $961.92 \mathrm{~cm}^{2}$ and $380.16 \mathrm{~cm}^{2}$ at layers of $10 \mathrm{~mm}$ and $30 \mathrm{~mm}$, respectively. However, at high measurement layers, the detectable area is decreased. The magnetic field spread out from the center and distributed over the surface of the considered area to provide a wider detected area. With the simulation results in Fig. 8 and measurement results in Fig. 12, we found that the pattern of reading status is in agreement with the $\mathrm{H}$ field simulation. Figure 11(d) illustrates an actual testing situation in laundry shop. The performance of the proposed magnetic field repeater and overall reader system is satisfied.

\section{Conclusion}

This paper presents a magnetic field shaping technique by using magnetic field repeater for HF-RFID system and near-field communications (NFCs) system. The performance of three cases is investigated: Case 1: the proposed magnetic field repeater, Case 2: the previous model [15] and Case 3: the off-the-shelf HF-RFID reader antenna. The proposed magnetic field repeater provides more detectable area than the previous model [15] and the off-theshelf HF-RFID reader in horizontal plane. However, at higher measurement layers, the detectable area is decreased. The magnetic field spread out from the center of reader antenna and distributed over the considered area. The proposed magnetic field shaping technique by using magnetic field repeater is suitable for improving a coverage area of the off-the-shelf HF-RFID reader in some applications such as laundry application, product tacking, etc. where a short range with a wide detected area are required. Moreover, an actual testing in laundry shop is performed. The performance of the proposed magnetic field repeater and the overall reader system is satisfied.

\section{Acknowledgments}

This work has been supported by the Thailand Research Fund MSD57I0034 and the TRF Senior Research Scholar Program with the contact number RTA6080008. The authors would like to thank the Silicon Craft Technology Co. Ltd. for HF-RFID reader.

\section{References}

[1] DOBKIN, D. M. The RF in RFID. Oxford, U.K.: Elsevier, 2008. ISBN: 9780080554020

[2] AL-OFEISHAT, H. A., RABABAH, M. A. A. A. Near field communication (NFC). International Journal of Computer Science and Network Security, 2012, vol. 12, no. 2, p. 97-99.

[3] LITEPOINT, A TERADYNE COMPANY. Test considerations for NFC enabled devices in manufacturing. White paper, 2016, 
p. 1-12. [Online] Available at: http://litepoint.com/wpcontent/uploads/2016/09/NFC-Whitepaper-090116.pdf

[4] NIKITIN, P. V., RAO, K. V. S., LAZAR, S. An overview of near field UHF-RFID. In Proc. of IEEE International Conference on RFID. Grapevine (TX, USA), 2007, p. 167-174. DOI: 10.1109/RFID.2007.346165

[5] WAFFENSCHMIDT, E. Wireless power for mobile devices. In 2011 IEEE 33rd International Telecommunications Energy Conference (INTELEC). Amsterdam (Netherlands), 2011, p. 1-9. DOI: 10.1109/INTLEC.2011.6099840

[6] Delphi Wireless Charging System. [Online]. Available at: http://delphi.com/

[7] UMENEI, A. E., SCHWANNECKE, J., VELPULA, S., et al. Novel method for selective nonlinear flux guide switching for contactless inductive power transfer. IEEE Transactions on Magnetics, 2012, vol. 48, no. 7, p. 2192-2195. DOI: 10.1109/TMAG.2012.2185246

[8] HUI, S. Y. R., HO, W. C. A new generation of universal contactless battery charging platform for portable consumer electronic equipment. In IEEE 35th Annual Power Electronics Specialists Conference. Aachen (Germany), 2004, p. 638-644. DOI: 10.1109/PESC.2004.1355823

[9] HUI, S. Y. R. Planar Inductive Battery Charger. UK patent application (GB2389720) and PCT patent application, Sep 2005.

[10] KIM, C. G., SEO, D.-H., YOU, J.-S., et al. Design of a contactless battery charger for cellular phone. IEEE Transactions on Industrial Electronics, 2001, vol. 48, no. 6, p. 1238-1247. DOI: $10.1109 / 41.969404$

[11] BUDHIA, M. COVIC, G. A., BOYS, J. T. Design and optimization of circular magnetic structures for lumped inductive power transfer systems. IEEE Transactions on Power Electronics, 2011, vol. 26, no. 11, p. 3096-3108. DOI: 10.1109/TPEL.2011.2143730

[12] AHN, D., HONG, S. A study on magnetic field repeater in wireless power transfer. IEEE Transactions on Industrial Electronics, 2013, vol. 60, no. 1, p. 360-371. DOI: 10.1109/TIE.2012.2188254

[13] PHAEBUA, K., LERTWIRIYAPRAPA, T., CHALERMWISUTKUL, S., et al. Area extension of a wireless battery charging system using multiple power repeater coil antennas. In 2nd International Conference on Intelligent Green Building and Smart Grid $(I G B S G) . \quad$ Prague (Czechia), 2016, p. 1-4. DOI: 10.1109/IGBSG.2016.7539438

[14] SAGHLATOON, H., MIRZAVAND, R., HONARI, M. M., et al. Investigation on passive booster for improving magnetic coupling of metal mounted proximity range HF-RFIDs. IEEE Transactions on Microwave Theory and Techniques, 2017, vol. 65, no. 9, p. 3401-3408. DOI: 10.1109/TMTT.2017.2676095

[15] SitTITHAI, P., LERTWIRIYAPRAPA, T., PHAEBUA, K. Study of multiple repeater antennas for high frequency (HF) RFID reader. In 2017 International Symposium on Antennas and Propagation (ISAP). Phuket (Thailand), 2017, p. 1-2. DOI: 10.1109/ISANP.2017.8229027

[16] SILICON CRAFT TECHNOLOGY Module Datasheet Pi-931$X A 5$. [Online] Available at: $\mathrm{http} / /$ www.sic.co.th/product.php?product $=152 \&$ category $=10$

[17] BALANIS, C. A. Antenna Theory: Analysis and Design. 3rd ed. New York: Wiley, 2005. ISBN: 978-0471667827

[18] SITTITHAI, P. Development of RFID system for industrial laundry business. Master Degree Thesis. King Mongkut's University of Technology North Bangkok, Thailand. 2017

[19] CST Microwave Studio, User's Manual, 2007.

\section{About the Authors ...}

Pinitnai SITTITHAI received his B.S. and M.S. Tech. Ed. in Electrical Engineering from King Mongkut's University of Technology North Bangkok, Thailand in 2014 and 2016, respectively.

Kittisak PHAEBUA received the B.Eng., M.Eng. degrees in Telecommunication Engineering, and Ph.D. (Eng.) degree in Electrical Engineering from King Mongkut's Institute of Technology Ladkrabang (KMITL), Bangkok, Thailand, in 2006, 2008 and 2012, respectively. He is currently an Assistant Professor at the Dept. of Teacher Training in Electrical Engineering, King Mongkut's University of Technology North Bangkok, Thailand. His research involves a computational in electromagnetic by using high frequency techniques, antenna and microwave filter design for wireless communication systems.

Titipong LERTWIRIYAPRAPA (corresponding author) received his B.S. Tech. Ed. in Electrical Engineering from King Mongkut's University of Technology North Bangkok in 1996 and his M.Eng. in Electrical Engineering from King Mongkut's Institute of Technology Ladkrabang in 2000. He received his M.Sc. and Ph.D. in Electrical Engineering from the Ohio State University, Columbus, Ohio, USA in 2006 and 2007, respectively. He is currently an Associate Professor at the Dept. of Teacher Training in Electrical Engineering, King Mongkut's University of Technology North Bangkok. He received the third place in the 2007 USNC/CNC URSI Student Paper Competition, held in Ottawa, Canada and the best paper award in the 2008 International Symposium on Antennas and Propagation (ISAP2008), held in Taiwan. He was the board committee of ECTI Association in 2012-2015. His research interests include electromagnetic theory, metamaterial, asymptotic, computational electromagnetics, and hybrid methods.

Prayoot AKKARAEKTHALIN received the B.Eng. and M.Eng. degrees in Electrical Engineering from King Mongkut's University of Technology North Bangkok (KMUTNB), Thailand, in 1986 and 1990, respectively, and the Ph.D. degree from the University of Delaware, Newark, USA, in 1998. From 1986 to 1988, he worked in the Microtek Co.Ltd., Thailand, as a microwave research and development engineer. In 1988, he joined the Dept. of Electrical and Computer Engineering KMUTNB, as instructor. His current research interests include passive and active microwave circuits, wideband and multiband antennas, telecommunications, and sensor systems. He published four books/book chapters and more than fifty papers in international journals. He is a member of IEEE, IEICE Japan, and ECTI Thailand. He was the Chairman for the IEEE MTT/AP/ED Thailand Joint Chapter during 2007 and 2008. He also served as the President for the ECTI Association, Thailand during 2012 and 2014 . He is currently the head of senior research scholar project granted by the Thailand Research Fund (TRF) during 2014-2020. 